\title{
Reclasificación de la severidad de la estenosis aórtica con la utilización del enfoque paraesternal derecho con transductor ciego
}

\author{
Dres. Álvaro Beltrán', Gustavo Galain¹, Marcos Pouso²
}

\begin{abstract}
Resumen foque paraesternal derecho (PED) es poco frecuente. go. res convencional y ciego. el PED reclasifica como severa una de cada dos.

Palabras clave: ESTENOSIS DE LA VÁLVULA AÓRTICA

ECOCARDIOGRAFÍA DOPPLER

TRANSDUCTORES
\end{abstract}

La severidad de la estenosis aórtica (EA) es uno de los pilares que decide la sustitución valvular. El ecocardiograma Doppler (ED) es de elección para el diagnóstico y cuantificación mediante la determinación de la velocidad máxima (VMáx), gradiente medio (GM) y área valvular (AV). VMáx y GM se obtienen desde múltiples enfoques, pero la utilización del en-

Objetivo: determinar la magnitud de la reclasificación de la EA con la utilización del enfoque PED con transductor cie-

Método: se estudian prospectivamente pacientes con EA moderada o severa por ED desde cualquier enfoque. Se considera EA moderada: VMáx 3 a 3,9 m/s, GM 20 a 39 mmHg o AV 1,1 a 1,5 cm². EA severa: VMáx $\geq 4$ m/s, GM $\geq 40$ mmHg o AV $\leq 1 \mathrm{~cm}^{2}$. Criterios de exclusión: fracción de eyección de ventrículo izquierdo (FEVI) $<50 \%$, insuficiencia valvular aórtica o mitral $\geq$ moderada, hemoglobina $(\mathrm{Hb})<11 \mathrm{~g} / \mathrm{dl}$. Se utilizan enfoques apical (ÁPEX), PED y subxifoideo con transducto-

Resultado: se incluyen 100 pacientes de $74,0 \pm 12$ años, sexo femenino $49 \%$, FEVI $69,1 \pm 8,4 \%$. Por ÁPEX EA severa $56 \%$ y no severa 44\%. Enfoque con gradiente más alto: ÁPEX 33/100, PED 48/100, más de un enfoque 19/100; las EA moderadas por ÁPEX son severas por PED: 18/33 (54,6\%, IC 95\% 36,4-71,9); leves por ÁPEX son severas por PED: 3/11 (27,3\% IC 95\% 6,0-61,0). PED reclasifica como severas 21 de las 44 consideradas moderadas o leves por ÁPEX (47,7\% IC 95\% 32,5-63,3).

Conclusión: el enfoque PED obtiene los valores de gradiente más altos en $48 \%$ de los casos. En EA no severa por ÁPEX,

\section{Reclassification of the severity of aortic stenosis obtaining gradients from right parasternal view with Pedof transducer}

\section{Summary}

The severity of aortic stenosis (AS) is one of the main issues for the decision of valve replacement. Doppler echocardiography (DE) is the modality of choice for diagnosis and quantification by maximum velocity (MV), medium gradient (MG), and valve area (VA). MV and MG can be obtained from multiple views, but the use of right parasternal (RPS) view is not frequent.

Objective: to know the magnitude of reclassification of AS with the use of right parasternal view with blind transducer. Method: patients with moderate or severe AS from any view by DE are prospectively studied. Moderate AS is considered with MV 3-3.9 m/s, MG 20-39 mmHg or VA 1.1-1.5 $\mathrm{cm}^{2}$. Severe AS: MV $\geq 4 \mathrm{~m} / \mathrm{s}, \mathrm{MG} \geq 40 \mathrm{~mm} \mathrm{Hg}$ or VA $\leq 1 \mathrm{~cm}^{2}$. Exclusion criteria: left ventricular ejection fraction $(\mathrm{LVEF})<50 \%, \geq$ moderate aortic or mitral valve regurgitation, hemoglobin $<$ $11 \mathrm{~g} / \mathrm{dl}$. Apical (ÁPEX), right para-sternal (RPS) and subxyfoid views are used with conventional and Pedof transducers.

1. Servicio de Ecografía Cardíaca (SEC), Montevideo. Uruguay.

2. Departamento Estadísticas y Métodos del Centro de Investigación Cardiovascular Uruguayo (CICU).

Correspondencia: Dr. Álvaro Beltrán. Correo electrónico: alvaro.beltran.uy@gmail.com

Recibido Set 21, 2017; aceptado Nov 17, 2017 
Results: We included 100 patients with age $74.0 \pm 12$ years, $49 \%$ women, LVEF $69.1 \pm 8.4 \%$. View with highest gradient: apex 33/100, RPS 48/100, more than one view 19/100; moderate by apex and severe by RPS: 18/33 (54,6\%, IC 95\% 36,4-71,9; mild by APEX and severe by RPS 3/11 (27.3\% IC 95\% 6.0-61.0). RPS reclassifies as severe 21 of the 44 evaluated as moderate or mild by ÁPEX (47.7\% IC95\% 32.5-63.3).

Conclusion: RPS obtains the highest gradients in $48 \%$ of patients. In non-severe AS by apical, RPS reclassifies as severe one out of two.

Key words: $\quad$ AORTIC VALVE STENOSIS DOPPLER ECHOCARDIOGRAPHY TRANSDUCERS

\section{Introducción}

Como consecuencia del incremento de la edad poblacional, la estenosis aórtica (EA) es actualmente la valvulopatía que con mayor frecuencia determina un reemplazo valvular. Cuando no se realiza oportunamente el tratamiento quirúrgico adecuado, el pronóstico es malo. La indicación quirúrgica se basa en la presencia de síntomas, en el compromiso de la fracción de eyección de ventrículo izquierdo (FEVI) y en la severidad de la estenosis. El ecocardiograma Doppler (ED) es la técnica de elección para el diagnóstico y la cuantificación de su severidad mediante la utilización de la velocidad máxima (VMáx), el gradiente medio (GM) y el área valvular (AV) medida por la ecuación de continuidad(1,2).

Para el diagnóstico preciso es fundamental una calidad óptima del registro de la señal Doppler. Esto se logra con la correcta alineación de las direcciones del chorro transvalvular y el haz de Doppler continuo (DC) y con buena relación señal/ruido. Esta depende de la distancia de la vena contracta (VC) al transductor, del tamaño del chorro en relación con el tamaño del haz de ultrasonido y de la sensibilidad del transductor ${ }^{(3)}$. La relación señal/ruido es baja en el adulto porque el chorro de estenosis está ubicado en el campo lejano del transductor, su tamaño es pequeño en relación con el del haz ultrasónico y la VC está próxima a la válvula calcificada, la cual genera ecos de gran amplitud que atenúan la señal del chorro. El transductor Pedof (DC sin imagen de eco 2D), al tener un cabezal más pequeño, permite una mejor penetración en los espacios intercostales, especialmente en la ubicación paraesternal derecha (PED). Por tener frecuencias más bajas que el transductor de eco $2 \mathrm{D}$, mejora además la relación señal/ruido.

La EA debe ser valorada desde diferentes enfoques $^{(4-6)}$, pero son muy escasos los trabajos diseñados para cuantificar su impacto ${ }^{(7,8)}$. Las Sociedades Europeas de Ecocardiografía y de Imagen Cardiovascular y la Sociedad Americana de Ecocardiografía aconsejan, para la valoración de la estenosis aórtica $^{(9,10)}$, la utilización del transductor Pedof cuando se interroga el enfoque PED.
La disminución del tiempo dedicado a cada estudio, la VMáx y GM registrados con el transductor de eco 2D desde el enfoque apical y la utilización excepcional de Pedof desde el enfoque PED en nuestro país, dificultan la correcta valoración de la EA. La mala alineación del haz ultrasónico con el chorro de sangre de la EA subvalora la VMáx y el GM, y sobrevalora el AV.

La finalidad de este trabajo es determinar la magnitud de la reclasificación de la EA con la utilización del enfoque PED con transductor ciego.

\section{Método}

\section{Población}

Entre marzo de 2012 y diciembre de 2016 se incluyeron en forma prospectiva y consecutiva 100 pacientes con diagnóstico por ED de EA moderada o severa, según los criterios de las guías de la Sociedad Europea de Cardiología (ESC)(1) y del Colegio Americano de Cardiología junto a la Asociación Americana del Corazón (ACC/AHA) ${ }^{(2)}$. Los criterios de EA moderada fueron: VMáx 3 a 3,9 m/s, GM 20 a $39 \mathrm{mmHg}$ o AV 1,1 a 1,5 cm². Se consideró EA severa si alguno de los siguientes criterios estaba presente desde cualquiera de los enfoques: VMáx $\geq 4 \mathrm{~m} / \mathrm{s}, \mathrm{GM}$ $\geq 40 \mathrm{mmHg}$ o $\mathrm{AV} \leq 1 \mathrm{~cm}^{2}$. Se excluyeron pacientes con FEVI $<50 \%$, insuficiencia valvular aórtica o mitral moderada o mayor ${ }^{(11,12)}, \mathrm{Hb}<11 \mathrm{~g} / \mathrm{dl}$, insuficiencia renal en hemodiálisis o intervención valvular previa.

\section{Protocolo de estudio}

A todos se les realizó un ED siguiendo las recomendaciones de la Sociedad Americana de Ecocardiografía $^{(9,10,13)}$. El diámetro de la cámara de salida del ventrículo izquierdo (CSVI) se midió con zoom en la mesosístole, de borde interno a borde interno, en paralelo y próximo al plano valvular aórtico. Para medir la velocidad de CSVI se utilizó el Doppler pulsado (DP) en el enfoque apical. Se ubicó la muestra de volumen próxima al lado ventricular de la válvula a una altura que permitiese el registro de un flujo laminar. La VMáx de la válvula aórtica con DC se 
Tabla 1. Datos generales de 100 pacientes con estenosis aórtica moderada o severa

\begin{tabular}{lc}
\hline Datos & \multicolumn{1}{c}{ Resultados } \\
\hline Edad & $74,0 \pm 12,0$ años \\
Sexo femenino & $49 / 100(49 \%)$ \\
ASC & $1,82 \pm 0,2 \mathrm{~m}^{2}$ \\
CSVI & $20,4 \pm 3 \mathrm{~mm}$ \\
\hline FEVI & $69,1 \pm 8,4 \%$ \\
\hline Presión arterial sistólica & $133,1 \pm 16 \mathrm{~mm} \mathrm{Hg}$ \\
\hline Presión arterial diastólica & $76 \pm 15,6 \mathrm{~mm} \mathrm{Hg}$ \\
\hline Asintomáticos & $53 / 100(53 \%)$ \\
\hline Ritmo sinusal & $87 / 100(87 \%)$ \\
Coronario conocido & $15 / 100(15 \%)$ \\
\hline HTA & $67 / 100(67 \%)$ \\
\hline Diabetes & $20 / 100(20 \%)$ \\
\hline Dislipemia & $36 / 100(36 \%)$ \\
\hline Tabaquismo & $5 / 100(5 \%)$ \\
\hline $\begin{array}{l}\text { ASC: área de superficie corporal; CSVI: diámetro de cámara de } \\
\text { salida del ventrículo izquierdo; Coronario conocido: paciente } \\
\text { con infarto agudo de miocardio, angioplastia coronaria o } \\
\text { puente coronario previos. }\end{array}$ \\
\hline
\end{tabular}

midió sobre el pico máximo de la curva, densa y bien dibujada, sin considerar ruidos y señales lineales finas. Con el trazado de la integral velocidad-tiempo sobre el borde externo de la señal densa se cuantificó el GM (figura 1). De forma rutinaria se estudiaron las ventanas apical, PED con Pedof (borde derecho del esternón en diferentes espacios intercostales con el paciente en decúbito lateral derecho) y subxifoidea. Se utilizaron filtros de pared altos y ganancias de DC bajas para definir nítidamente las curvas Doppler que se desplegaron en la pantalla a velocidades de barrido de $100 \mathrm{~mm} / \mathrm{s}$. Para la obtención de la curva se tomó en cuenta la nitidez de los bordes, la intensidad de la señal y las características acústicas.

La VMáx y el GM se definieron como el valor más alto obtenido desde cualquiera de los enfoques cuando la diferencia de las mediciones era mayor a 2DS para nuestra variabilidad interobservador: $\geq 0,2 \mathrm{~m} / \mathrm{s}$ para VMáx y $\geq 4 \mathrm{mmHg}$ para $\mathrm{GM}^{(14)}$. La FEVI se midió con el método Simpson biplano. Cuando el paciente estaba en ritmo sinusal cada medida se obtuvo promediando las obtenidas en tres latidos y en fibrilación auricular cinco latidos, preferentemente con frecuencias entre 60 y 90 contracciones por minuto. A todos los pacientes se les midió la tensión arterial inmediatamente después
Tabla 2. Enfoques en que se registran los máximos valores de VMáx y GM

\begin{tabular}{lc}
\hline Enfoques & Resultados \\
\hline Enfoque ÁPEX & $33 / 100(33 \%)$ \\
Enfoque PED & $48 / 100(48 \%)$ \\
$>$ de un enfoque & $19 / 100(19 \%)$ \\
\hline
\end{tabular}

de terminado el registro de DC de la válvula aórtica. Si la tensión arterial era superior a $160 / 100 \mathrm{mmHg}$ se volvía a realizar el estudio una vez normalizada la misma.

Se utilizaron equipos HP7500, HP5500 y GE Vivid I con transductores convencionales y Pedof (pequeños, doble cristal, frecuencia de $1.9 \mathrm{MHz}$ y con alta relación señal/ruido). Los estudios se almacenaron digitalmente para el análisis offline.

\section{Análisis estadístico}

Los valores categóricos se expresaron como porcentaje, los continuos como medias \pm desvío estándar (DE); las diferencias de las medias se analizaron con el test $\mathrm{t}$ de Student y los intervalos de confianza exactos se calcularon mediante el método de Fisher.

\section{Resultados}

En forma prospectiva y consecutiva se enrolaron 100 pacientes con una edad media de 74,0 $\pm 12,0$ años, $49 \%$ de sexo femenino, $47 \%$ asintomáticos y $87 \%$ en ritmo sinusal con una FEVI media de $69,1 \%$ $\pm 8,4 \%$, presión arterial sistólica de $133,1 \pm 16$ mmHg y diastólica de $76 \pm 15,6 \mathrm{mmHg}$ (tabla 1 ).

No se encontraron diferencias estadísticamente significativas en las VMáx y GM obtenidos desde ÁPEX y PED entre los pacientes en FA y en ritmo sinusal.

La factibilidad del enfoque apical fue de $97 \%$ y la de PED 75\%. Los valores más altos de VMáx y GM se obtuvieron en el enfoque apical en 33/100 (33\%), en PED 48/100 (48\%) y en más de un enfoque en 19/100 (19\%) (tabla 2). En el 3\% en el enfoque subxifoideo se obtuvieron valores similares al apical o PED.

Las medias de VMáx y de GM fueron más altas en el enfoque PED que en el apical. La VMáx media en ápex fue 4,09 $\pm 0,6 \mathrm{~m} / \mathrm{s}$ y en PED $4,3 \pm 0,6 \mathrm{~m} / \mathrm{s}$ (p $<0,05$ ), en tanto el GM apical fue $40,3 \pm 0,7 \mathrm{mmHg}$ $\mathrm{y}$ en PED 45,0 \pm 0,7 mmHg $(\mathrm{p}<0,05)$ (tabla 3 ).

Cuando se catalogó la EA solamente por ÁPEX (tabla 4), 56 fueron clasificadas como severas y 44 como no severas (33 como moderadas y 11 como leves). La utilización del enfoque PED reclasificó co- 
Tabla 3. Promedio de medidas de VMáx, GM y AV

\begin{tabular}{lcc}
\hline V Máx en ÁPEX & $4,09 \pm 0,6 \mathrm{~m} / \mathrm{s}$ & $\mathrm{p}<0,05$ \\
V Máx en PED & $4,3 \pm 0,6 \mathrm{~m} / \mathrm{s}$ & \\
GM en ÁPEX & $40,3 \pm 0,7 \mathrm{mmHg}$ & $\mathrm{p}<0,05$ \\
GM en PED & $45,0 \pm 0,7 \mathrm{mmHg}$ & \\
AV por ÁPEX & $0,89 \pm 0,27 \mathrm{~cm}^{2}$ & p NS \\
AV por PED & $0,86 \pm 0,26 \mathrm{~cm}^{2}$ & \\
\hline
\end{tabular}

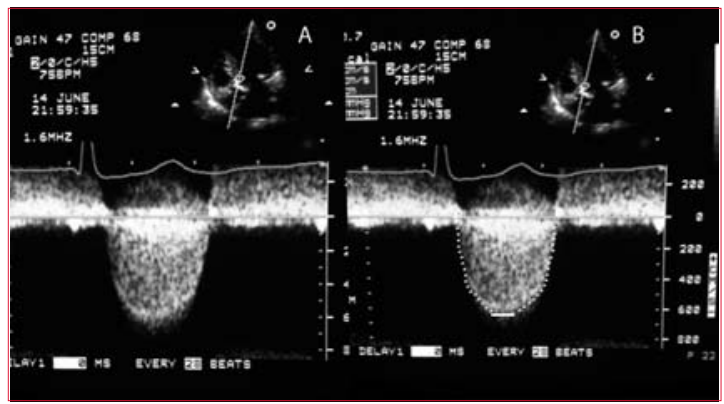

Figura 1. Registro con DC desde el enfoque apical. A: Registro sobre el que se realizan las mediciones. B: La VMáx se mide sobre el pico máximo de la curva, densa y bien dibujada, sin considerar los ruidos y las señales lineales finas. El trazado de la integral velocidad-tiempo se realiza sobre el borde externo de la envolvente, densa y bien definida.

mo severas 18/33 (54,6\%, IC 95\% 36,4-71,9) que eran moderadas por ÁPEX (figura 1) y $3 / 11(27,3 \%$ IC 95\% 6,0-61,0) leves por ÁPEX (figura 2) . En total $21 / 44(47,7 \%)$ de las EA no severas por ÁPEX fueron reclasificadas como severas por PED, es decir uno de cada dos pacientes.

La EA muy severa con VMáx $\geq 5 \mathrm{~m} / \mathrm{s}$ se registró en 15/100. En ÁPEX se obtuvo en 4/15 (26,7\%), PED en 7/15 (46,7\%) y en más de un enfoque en $4 / 15$ (26,7\%). Entre las EA asintomáticas y severas por ÁPEX, 3/39 (7,7\%) fueron reclasificadas por PED como EA muy severas.

\section{Discusión}

Este trabajo demuestra que utilizar solo el enfoque apical no diagnostica una de cada dos EA severas, valoradas como leves o moderadas por ÁPEX. Uno de los obstáculos en el manejo clínico de los pacientes con EA es la correcta valoración de la severidad de la valvulopatía. Ella explica la causa de los síntomas y ayuda a establecer el momento óptimo de la sustitución valvular.

Las guías establecen como criterios de severidad de la EA: VMáx $\geq 4 \mathrm{~m} / \mathrm{s}$, gradiente medio $\geq 40 \mathrm{mmHg}$
Tabla 4. Grados de severidad de la estenosis aórtica desde los diferentes enfoques.

\begin{tabular}{lc}
\hline Severidad de la EA & Resultados \\
\hline EA severa por ÁPEX & $56 / 100(56 \%)$ \\
EA no severa por ÁPEX & $44 / 100(44 \%)$ \\
EA moderada por ÁPEX & $18 / 33(54,5 \%)$ \\
y severa por PED & IC $95 \% 36,4-71,9$ \\
EA leve por ÁPEX & $3 / 11(27,3 \%)$ \\
y severa por PED & IC $95 \% 6,0-61,0$ \\
\hline
\end{tabular}

o área valvular $\leq 1 \mathrm{~cm}^{2(1,2)}$. Las mediciones de las velocidades, GM y la CSVI tienen variaciones en las medidas que se multiplican en la ecuación de continuidad del AV con el consiguiente arrastre de los errores. En esta población se excluyeron las situaciones que podían aumentar el flujo transvalvular (anemia, regurgitaciones, fístulas arteriovenosas) y las que podían disminuirlo (compromiso de la FEVI). Se tomó el valor más alto entre los enfoques cuando era superior a 2 DS de nuestra variabilidad interobservador ${ }^{(14)}$. Como el diámetro y la velocidad de la CSVI no varían si se considera uno u otro enfoque y se minimiza la variación del flujo valvular, la VMáx y el GM dependen directamente de la severidad de la EA. Su valoración requiere especial cuidado en los aspectos técnicos. La causa más frecuente de error es subestimar la VMáx y el GM por no alinear en forma paralela el haz del DC con la dirección del chorro estenótico. Nosotros coincidimos con la reciente recomendación del uso de Pedof desde múltiples enfoques ${ }^{(9,10)}$. La disminución del tiempo dedicado a cada estudio en nuestro país, el menor entrenamiento en obtener imágenes desde el PED y la utilización del transductor de eco $2 \mathrm{D}$ con $\mathrm{DC}$ llevan a que habitualmente la EA solo se valore desde el enfoque apical.

Una de las primeras publicaciones que llama la atención sobre el enfoque PED en la $\mathrm{EA}^{(4)}$, señala que tiene una factibilidad de solo $49 \%$, pero con mejor correlación con la hemodinamia que el enfoque apical. Otto ${ }^{(6)}$, en el trabajo de seguimiento de EA, considera los enfoques PED y supraesternal juntos y obtiene los valores más altos de VMáx y GM en $33 \%$ de los casos. De Monchy ${ }^{(7)}$ utiliza únicamente el AV como criterio de severidad y describe un error de clasificación entre ápex y PED de 21\% (17\% de leve a moderada y $4 \%$ de moderada a severa). Thaden ${ }^{(8)}$, que también utiliza criterios de severidad por AV, describe una factibilidad del PED de $81 \%$ con obtención de los valores más altos en el $50 \%$. Si solo se utiliza el enfoque apical hay $23 \%$ de error. En nuestra población se considera EA severa cuando, 

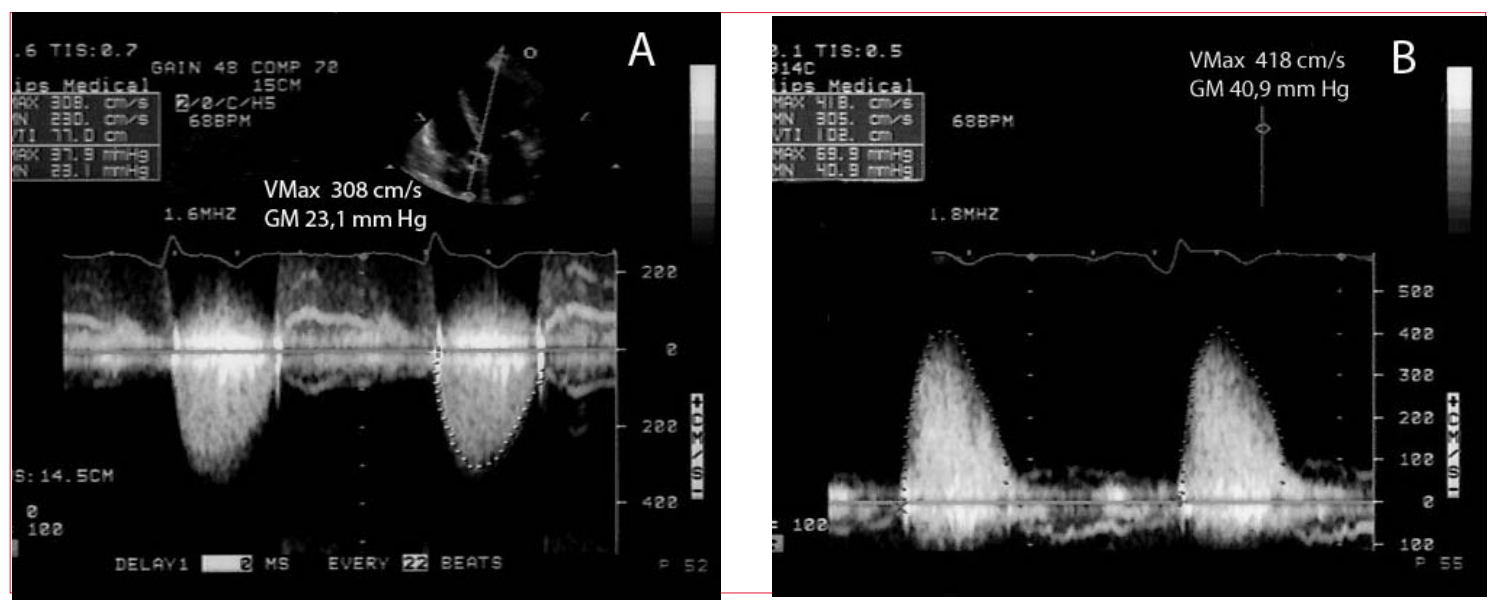

Figura 2. Registros desde APEX y PED en el mismo paciente. A: APEX con valores de VMáx y GM que catalogan la EA como leve. B: PED con Pedof con VMáx y GM correspondientes a EA severa.
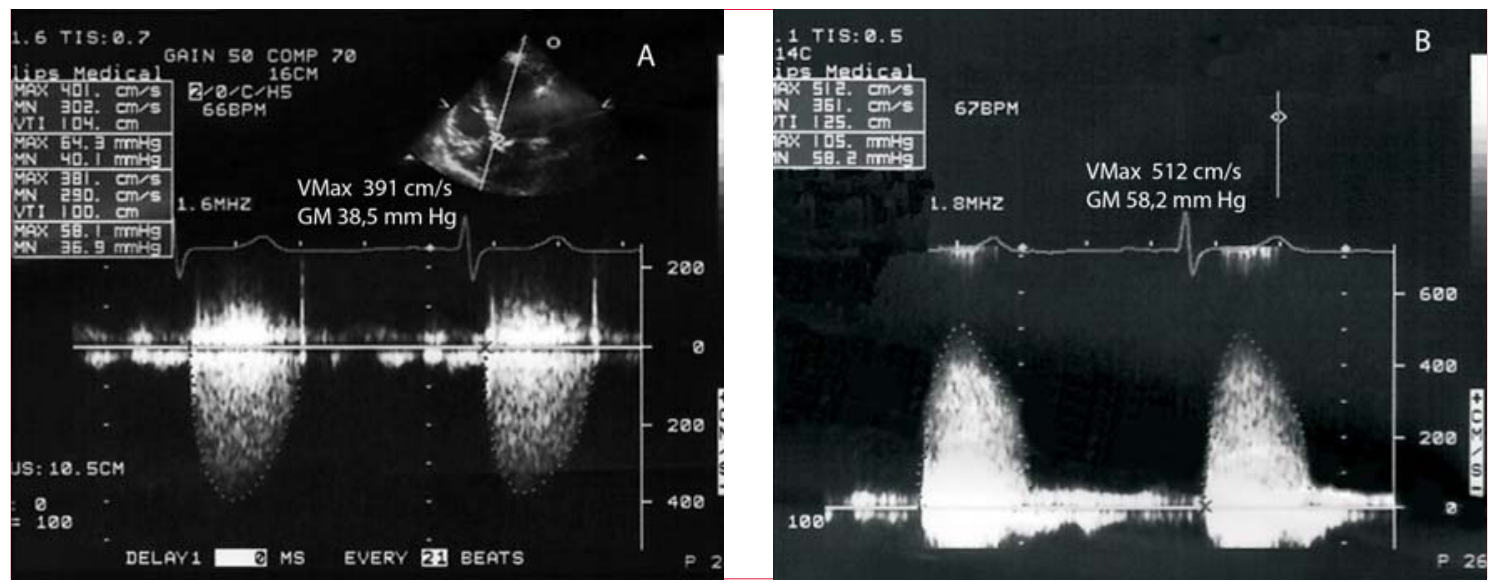

Figura 3. Registros desde ÁPEX y PED en el mismo paciente. A: ÁPEX con valores de VMáx y GM de EA moderada. B: PED con Pedof con VMáx y GM correspondientes a EA muy severa.

desde cualquier enfoque, está presente uno de los tres criterios: VMáx, GM o AV. En EA no severa por ÁPEX fueron reclasificadas como severas por PED $21 / 44(47,7 \%)$.

Las guías sobre manejo de la enfermedad valvular de la AHA/ACC ${ }^{(2)}$ recomiendan como indicación clase IIA la sustitución valvular aórtica en la EA severa asintomática con FEVI conservada y VMáx $\geq 5$ $\mathrm{m} / \mathrm{s}$, en tanto la ESC la aconseja con VMáx $\geq 5,5 \mathrm{~m} / \mathrm{s}$ (1). En un trabajo reciente, Zilberszac ${ }^{(15)}$ realiza un seguimiento de 103 pacientes mayores de 70 años con EA severa asintomática. Define la EA muy severa con una VMáx $\geq 5 \mathrm{~m} / \mathrm{s}$ en base a que es un punto de corte que predice la evolución en forma independiente. La sobrevida libre de eventos de la EA con VMáx entre $4 \mathrm{~m} / \mathrm{s}$ y $5 \mathrm{~m} / \mathrm{s}$ comparada con $\geq 5 \mathrm{~m} / \mathrm{s}$ a un año es de $84 \%$ y $57 \%$ y a los dos años de $57 \%$ y $21 \%$, respectivamente.

En el presente estudio la EA asintomática y severa por ÁPEX es reclasificada por PED como muy severa en $3 / 39(7,7 \%)$.
En el informe ecocardiográfico debe consignarse específicamente desde qué enfoque se obtienen las velocidades y gradientes máximos. Estos datos serán fundamentales para el seguimiento clínico del paciente. En los sucesivos ED de control, si no se especifica desde qué enfoque se obtienen las medidas, sus variaciones pueden interpretarse como una rápida progresión de la $\mathrm{EA}$, con la consiguiente indicación errónea de sustitución valvular.

\section{Conclusiones}

La utilización rutinaria del enfoque $\mathrm{PED}$ es mandatoria en todo estudio de EA. La VMáx y el GM de mayor valor se obtienen en el $48 \%$ de los casos desde el PED. Esto lleva a que prácticamente una de cada dos EA no severa por ÁPEX, sea severa por PED. En la EA asintomática severa por ÁPEX, el PED reclasifica como muy severa el 7,7\%. En el seguimiento clínico de la EA, el informe ecocardiográfico debe 
consignar específicamente desde qué enfoque se obtienen VMáx y GM.

\section{Bibliografía}

1. Baumgartner H, Falk V, Bax JJ, De Bonis M, Hamm C, Holm PJ, et al. 2017 ESC/EACTS Guidelines for the management of valvular heart disease. Eur Heart J 2017; 38(36):2739-91.

2. Nishimura RA, Otto CM, Bonow RO, Carabello BA, Erwin JP 3rd, Guyton RA, et al. 2014 AHA/ACC Guideline for the Management of Patients With Valvular Heart Disease: a report of the American College of Cardiology/American Heart Association Task Force on Practice Guidelines. Circulation 2014; 129(23):e521-643.

3. Weyman AE. Principles and practice of echocardiography. 2 ed. Pennsylvania: Lea \& Febiger, 1994:163-83.

4. Williams GA, Labovitz AJ, Nelson JG, Kennedy HL. Value of multiple echocardiographic views in the evaluation of aortic stenosis in adults by continuous-wave Doppler. Am J Cardiol 1985; 55(4): 445-9.

5. Tavli T, Ammar A, Wong M. Doppler-derived aortic valve gradients: imaging versus non-imaging techniques. J Heart Valve Dis 1993; 2(3):253-6.

6. Otto CM, Burwash IG, Legget ME, Munt BI, Fujioka M, Healy NL, et al. Prospective study of asymptomatic valvular aortic stenosis: clinical, echocardiographic, and exercise predictors of outcome. Circulation 1997; 95(9):2262-70.

7. de Monchy CC, Lepage L, Boutron I, Leye M, Detaint D, Hyafil F, et al. Usefulness of the right parasternal view and non-imaging continuous-wave Doppler transducer for the evaluation of the severity of aortic stenosis in the modern area. Eur J Echocardiogr 2009; 10(3):420-4.

8. Thaden JJ, Nkomo VT, Lee KJ, Oh JK. Doppler Imaging in aortic stenosis: the importance of the nonapical imaging windows to determine severity in a contemporary cohort. J Am Soc Echocardiogr 2015; 28(7):780-5.
9. Baumgartner H, Hung J, Bermejo J, Chambers JB, Evangelista A, Griffin BP, et al. Echocardiographic assessment of valve stenosis: EAE/ASE recommendations for clinical practice. J Am Soc Echocardiogr 2009; 22(1):1-23

10. Baumgartner H Chair, Hung J Co-Chair, Bermejo J, Chambers JB, Edvardsen T, Goldstein $\mathbf{S}$, et al. Recommendations on the echocardiographic assessment of aortic valve stenosis: a focused update from the European Association of Cardiovascular Imaging and the American Society of Echocardiography. Eur Heart J Cardiovasc Imaging 2017; 18(3):254-275.

11. Lancellotti P, Tribouilloy C, Hagendorff A, Moura L, Popescu BA, Agricola E, et al. European Association of Echocardiography recommendations for the assessment of valvular regurgitation. Part 1: aortic and pulmonary regurgitation (native valve disease). Eur J Echocardiogr 2010; 11(3): 223-44.

12. Lancellotti P, Moura L, Pierard LA, Agricola E, Popescu BA, Tribouilloy C, et al. European Association of Echocardiography recommendations for the assessment of valvular regurgitation. Part 2: mitral and tricuspid regurgitation (native valve disease). Eur J Echocardiogr 2010; 11(4):307-32.

13. Lang RM, Bierig M, Devereux RB, Flachskampf FA, Foster E, Pellikka PA, et al. Recommendations for chamber quantification: a report from the American Society of Echocardiography's Guidelines and Standards Committee and the Chamber Quantification Writing Group, developed in conjunction with the European Association of Echocardiography, a branch of the European Society of Cardiology. J Am Soc Echocardiogr 2005; 18(12): 1440-63.

14. Beltrán A, Galain G, Pouso M. Reproducibilidad y variabilidad de las medidas ecocardiográficas en la estenosis valvular aórtica. Rev Urug Cardiol 2015; 30(supl 1):S40.

15. Zilberszac R, Gabriel H, Schemper M, Laufer G, Maurer G, Rosenhek R. Asymptomatic Severe Aortic Stenosis in the Elderly. JACC Cardiovasc Imaging $2017 ; 10(1): 43-50$. 